Supplementary information

\title{
Selective killing of Shiga toxin-producing Escherichia coli with antibody-conjugated chitosan nanoparticles in the gastrointestinal tract
}

\author{
Zhengxin Ma ${ }^{1,2}$, Minyoung Kang ${ }^{1,2}$, Shanyu Meng 3 , Zhaohui Tong 3 , Soon-Do Yoon ${ }^{4}$, \\ Yeongseon Jang ${ }^{5}$ and K. Casey Jeong ${ }^{1,2^{*}}$
}

${ }^{1}$ Emerging Pathogens Institute, University of Florida, Gainesville, Florida 32611, USA

${ }^{2}$ Department of Animal Sciences, University of Florida, Gainesville, Florida 32611, USA

${ }^{3}$ Department of Agricultural and Biological Engineering, University of Florida, Gainesville, Florida 32611, USA

${ }^{4}$ Department of Chemical and Biomolecular Engineering, Chonnam National University, Yeosu, Jeonnam 550-749, Republic of Korea

${ }^{5}$ Department of Chemical Engineering, University of Florida, Gainesville, Florida 32611, USA

${ }^{*}$ Corresponding author:

K. Casey Jeong, $\mathrm{PhD}$

Associate Professor of Microbiology

Emerging Pathogens Institute

Department of Animal Sciences

University of Florida

2055 Mowry Rd

Gainesville, FL 32611

E-mail: kcjeong@ufl.edu

Phone: 1-352-294-5376 


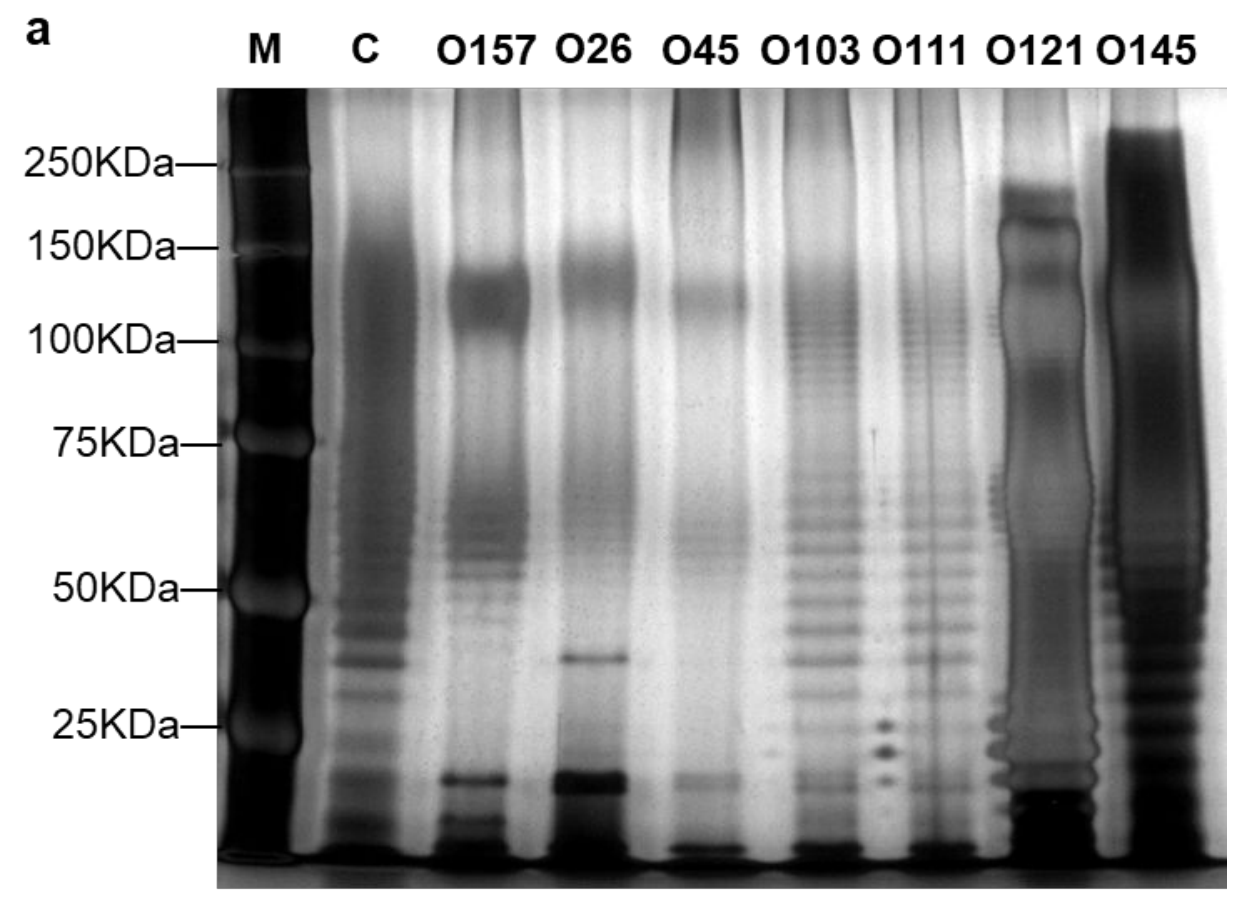

b

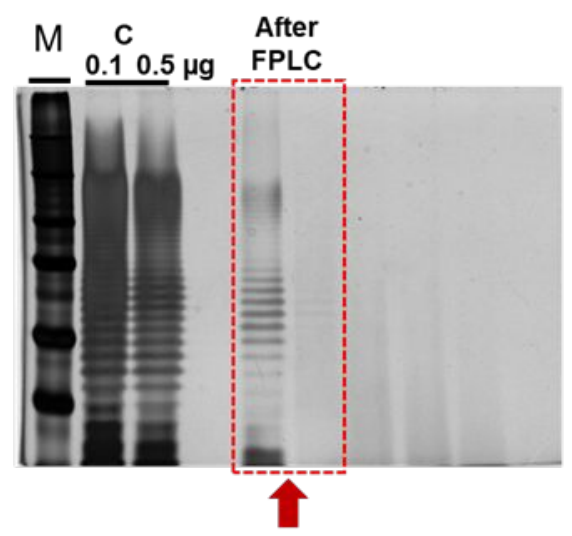

C

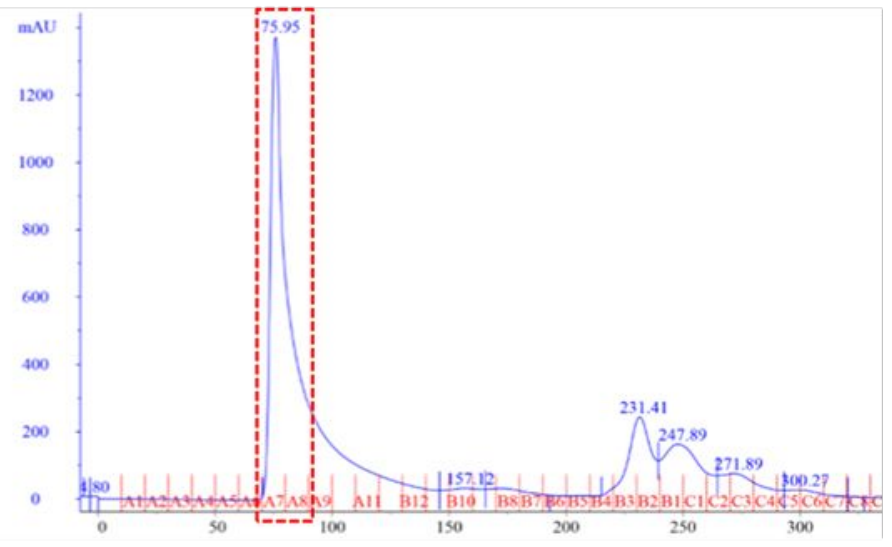

Figure S1. Extraction and purification of LPS of STEC. a Crude LPS of 7 STEC strains was extracted by a hot-phenol-water extraction method and subjected on SDS-PAGE. M: Protein 
marker, C: positive control (commercially available LPS of E. coli O111:B4), and extracted LPS from each serotype (O157, O26, O45, O103, O111, O121, and O145) are listed on top of the gel. b Crude LPS samples were purified by fast protein liquid chromatography (FPLC) and fractions were confirmed by SDS-PAGE gel electrophoresis. O-side chain fraction from E. coli $\mathrm{O} 103$ is shown as a representative. C; positive control (LPS of E. coli O111:B4). c A chromatogram of FPLC fraction of E. coli O103. O-side chain fraction (boxed in dashed square) of E. coli $\mathrm{O} 103$ was collected and analyzed by SDS-PAGE as shown in $\mathbf{b}$.

Table S1. Yields of IgY antibodies after immunization with antigens from each serotype.

\begin{tabular}{lll}
\hline E. coli serotype & Egg numbers & IgY total yield $(\mathrm{g})$ \\
\hline O26 & 139 & 10.89 \\
O45 & 138 & 11.22 \\
O103 & 141 & 11.67 \\
O111 & 126 & 9.88 \\
O121 & 130 & 9.92 \\
O145 & 141 & 10.96 \\
O157 & 140 & 11.01 \\
\hline
\end{tabular}

